\title{
Increasing access to brief Coping Strategy Enhancement for distressing voices: a service valuation exploring a possible role for briefly-trained therapists - CORRIGENDUM
}

Phil Clarke, Anna-Marie Jones and Mark Hayward

https://doi.org/10.1017/S1754470X21000143, Published by Cambridge University Press, 23 June 2021.

Keywords: CBT; coping; hallucinations; psychotherapy training; voice-hearing; corrigendum

In this item the title was published incorrectly. The word 'valuation' should be 'evaluation'.

The correct title is 'Increasing access to brief Coping Strategy Enhancement for distressing voices: a service evaluation exploring a possible role for briefly-trained therapists'.

\section{Reference}

Clarke, P., Jones, A.-M. \& Hayward, M. Increasing access to brief Coping Strategy Enhancement for distressing voices: a service valuation exploring a possible role for briefly-trained therapists. The Cognitive Behaviour Therapist 14. https://doi. org/10.1017/S1754470X21000143

Cite this article: Clarke P, Jones A-M, and Hayward M. Increasing access to brief Coping Strategy Enhancement for distressing voices: a service valuation exploring a possible role for briefly-trained therapists - CORRIGENDUM. The Cognitive Behaviour Therapist. https://doi.org/10.1017/S1754470X21000209

(C) The Author(s), 2021. Published by Cambridge University Press on behalf of the British Association for Behavioural and Cognitive Psychotherapies 